\title{
The Usability and Effectiveness of Interactive Video as a Complementary Child Pedestrian Training Activity
}

\author{
James A. Hammond, Tom J. Cherrett, and Ben J. Waterson
}

\begin{abstract}
Practical on-street pedestrian training is one form of education designed to promote safe roadside behavior amongst young road users with the intention of reducing their potential of involvement in road traffic collisions. While many local authorities in the United Kingdom operate pedestrian training, a number are reducing the amount of on-street training on offer as a result of financial and time constraints. This reduction in practical training puts more emphasis on paper-based classroom activities which increase knowledge acquisition but are generally not as effective in improving practical skills. Interactive videos may prove to be an effective complementary activity alongside pedestrian training as it is suggested that they can more effectively target hard skills compared to paper-based activities. This paper evaluates the effectiveness of an interactive road safety video designed to improve children's crossing skills between parked cars when no other alternative safer route is available. The paper finds that interactive video shows the potential to improve the crossing behavior of children and that it therefore may make a useful additional educational activity alongside pedestrian training.
\end{abstract}

Index Terms-E-learning, interactive video, pedestrian training, road safety.

\section{INTRODUCTION}

Road safety is a global public health issue with approximately 1.2 million people being killed on roads as a result of road traffic collisions in 2010 [1].

Ingrained in a systems approach to improving the road safety situation; Engineering, Enforcement, Education and Evaluation are often used to reduce the likelihood and severity of collisions which, alongside other system elements such as post-crash response and critical care have contributed to substantial reductions in deaths in many countries [1]. Despite much success there is still great global spatial variation in death and injury rates as a result of road traffic collisions [1]. The United Kingdom has made substantial overall progress in reducing the number of people killed and seriously injured on its roads however there is still more that can be done to address the issue. One area of concern is child pedestrians that are injured on roads and with over 2000 5-15 year old pedestrians reported to be involved in a traffic related injury when walking to school in 2010 in the UK there

Manuscript received May 24, 2013; revised July 22, 2013. This work was supported in part by the Engineering and Physical Sciences Research Council in the United Kingdom.

The authors are with the Transportation Research Group at the University of Southampton, Hampshire, United Kingdom (e-mail: j.hammond@soton.ac.uk). is scope to do more to address the issue [2].

Education, Training and Publicity are key measures used in the United Kingdom to raise awareness of child road safety issues, increase safety knowledge and to develop safe roadside skills. In this paper we investigate how an e-learning based interactive video could be used to improve child pedestrian skills and act as a complementary educational material to run alongside existing practical pedestrian training to improve behavior and reduce pedestrian-vehicle conflicts and collisions.

\section{BACKGROUND}

Pedestrian training forms one educational measure implemented in the United Kingdom specifically targeting pedestrian skills and roadside awareness [3] with the aim of improving skills and safety and therefore reducing the likelihood of conflict and subsequent road traffic collisions and injuries.

Government advocated training generally offers practical experience and coaching in three key areas; finding safe routes and places to cross the road, how to cross safely at junctions and how to cross safely between parked cars when a safer alternative does not exist [4]. A notable practical pedestrian training scheme, Kerbcraft, was trialed from 2002-2007 and was found to statistically improve roadside skills on-street following training [5]. Recent evidence indicates that few Local Authorities are implementing Kerbcraft fully and are instead offering less comprehensive, adapted schemes, largely based on the original scheme but with less practical roadside training elements [6].

Local Authorities are as a result often unable to offer training in all skill areas, in particular crossing between parked cars either due to time constraints, financial constraints or where a lack of suitable, safe training locations exist. Despite the risk of crossing between parked cars, especially for children where driver-to-pedestrian sight-lines are compromised, there are some instances where safer crossing locations do not exist and therefore a safe procedure for crossing is necessary [7]. In cases where crossing between parked cars is not demonstrated as part of a practical pedestrian training program, paper based or 'chalk-and-talk' activities are often substituted. There is evidence to suggest that paper based activities are less able to transfer knowledge into roadside behavior when compared to practical training [6], [8].

Interactive health and safety training videos have shown potential to impart 'hard' skills [9]. Cherrett, Wills et al. [9] demonstrated that a transportation surveying interactive health and safety video offered an engaging addition to lecture materials and that the principals could be applicable to other 'hard' procedural activities such as those required for 
crossing roads. In the video, users were asked to watch a video of risky behavior related to setting up a roadside survey using camera equipment. The player had to highlight dangers and answer multiple choice questions before having the safe procedure demonstrated. Similarly, a hazard perception based cycling interactive video was developed and intended to be a demonstration of EU best practice, outlining the potential for interactive video to be a suitable educational aid in improving road users' hazard awareness and skills [10]. The video relied on users' hazard perception skills, requiring them to identify cycling hazards, such as vehicles encroaching onto a cycle lane, in order to progress through the interactive video (Fig. 1). While the video was developed and tested, it was not evaluated regarding its impact on behavior [10]; a critical measure of the success of the video. Further to this, a pilot study of an interactive video aimed at improving the safety of children when crossing between parked cars demonstrated potential to transfer multisensory audio-visual information presented on a computer, in the form of an interactive video, into behavior at the roadside [11]. This transfer of information from a computer to behavior at the roadside is an important feature that does not often take place from paper based materials.

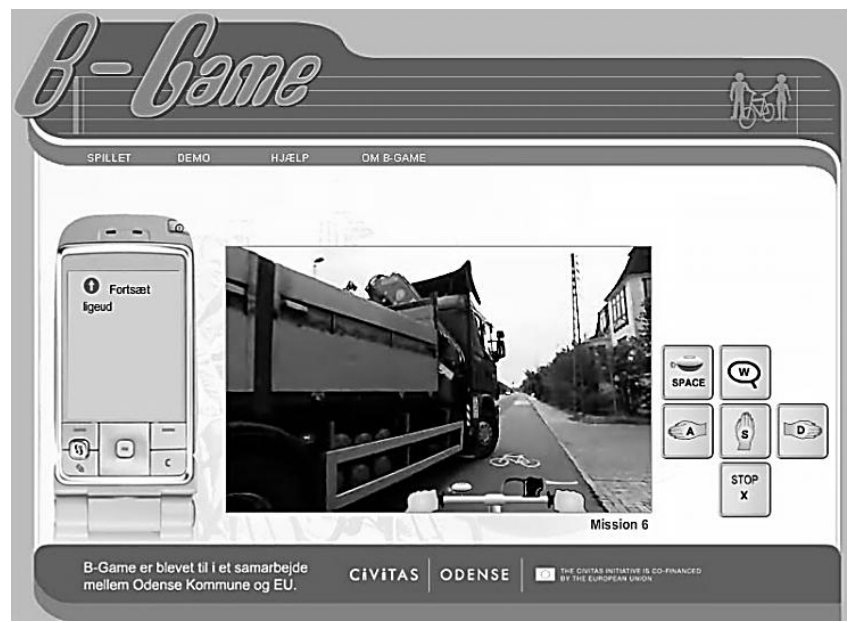

Fig. 1. B-Game cycling road safety interactive video [10]

This paper seeks to build upon the pilot study to further establish the effectiveness of a road safety interactive parked cars crossing video in improving roadside behavior, as well as offering design guidance for these types of videos, should they be developed as an additional road safety training aid for pedestrian training schemes. We hypothesize that interactive road safety videos can engage users to the extent that behavior change can occur as a result of the intervention which when used alongside pedestrian training schemes should act to reinforce the content delivered.

While in no way is this suggested to be a replacement for practical pedestrian training, interactive road safety videos could be used as a supportive or complementary training material alongside practical training and other road safety classroom activities.

\section{MeThodology}

\section{A. Developing the Interactive Road Safety Video}

An interactive video was designed to impart knowledge, to users in the age range of 5-7, on how to cross safely between parked cars when no other safer alternative exists with a focus on behavior transfer and roadside skill improvement. The skill was selected on the grounds that a) in some school locations, other factors, for example a busy road, prevent practical training taking place and b) many residential roads are lined with parked cars and thus the skill may be essential to some users despite the inherent risks.

Based around the premise of hazard perception and identification, the interactive video requires users to identify hazards in the parked cars crossing procedure of two young road users. Each hazard is hot-spotted, allowing a user to select a hazard directly on the video in order to stimulate engagement in the video. A hot-spot (Fig. 2) is defined here as a specific user selectable video region, active for a specific period of time with the active region relocating in progression with the location of the target behavior. For example, a video of a child running across the road would be hot-spotted such that the behavior is user selectable as the child progresses through the dangerous crossing, while the remainder of the video remains inactive.

The video interface allows the interactive video to be paused and rewound at any time (Fig. 3(a)) such that a user can identify hazards in their own time. To aid as a hint to young users, the mouse cursor changes to a hand symbol accompanied by a hazard symbol upon moving the mouse over a hazard (Fig. 3(b)). The correct identification of a hazardous behavior is awarded with a scoreboard point (Fig. 3(c)) and immediately followed by spoken instructions on how to perform the highlighted hazard in a safe manor (Fig. 3(d)). The users get two opportunities to identify the hazards.

(a)

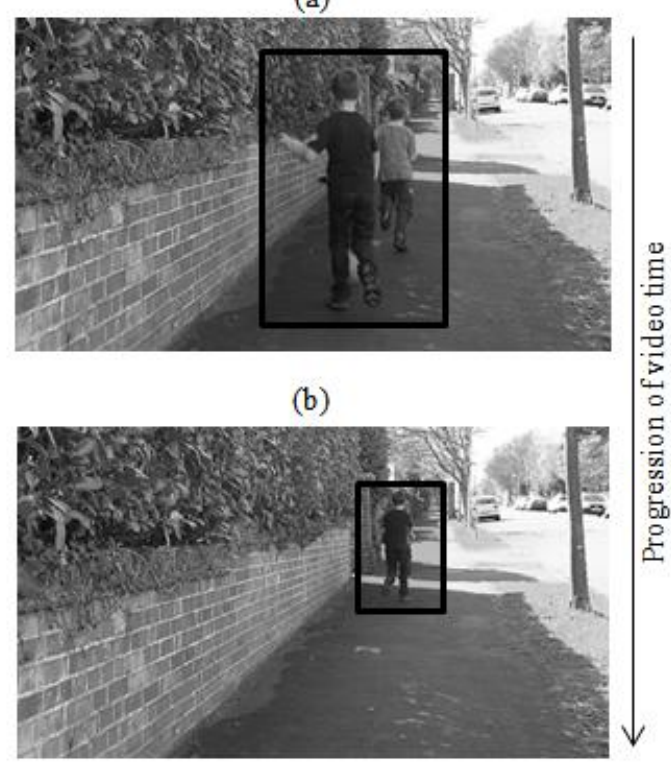

Fig. 2. The hot-spot concept.

Incorrect identifications are not penalized as the task has the primary purpose of stimulating the user and encouraging engagement in the video with the aim of improving procedural behavior rather than the underlying hazard awareness skills of users. The interactive video is preceded by an instructional video explaining its use and is followed at the end by two further repetitions of the correct, crossing safely between parked cars procedure; once in a user-paced step-wise sequence, such that each part of the crossing 
sequence can be seen independently with the users advancing between steps in their own time and once as a full crossing sequence so that users are aware of the full procedure in context.

The highlighted hot-spotted area is user selectable and importantly moves with the hazard as the video progresses from (a) to (b).
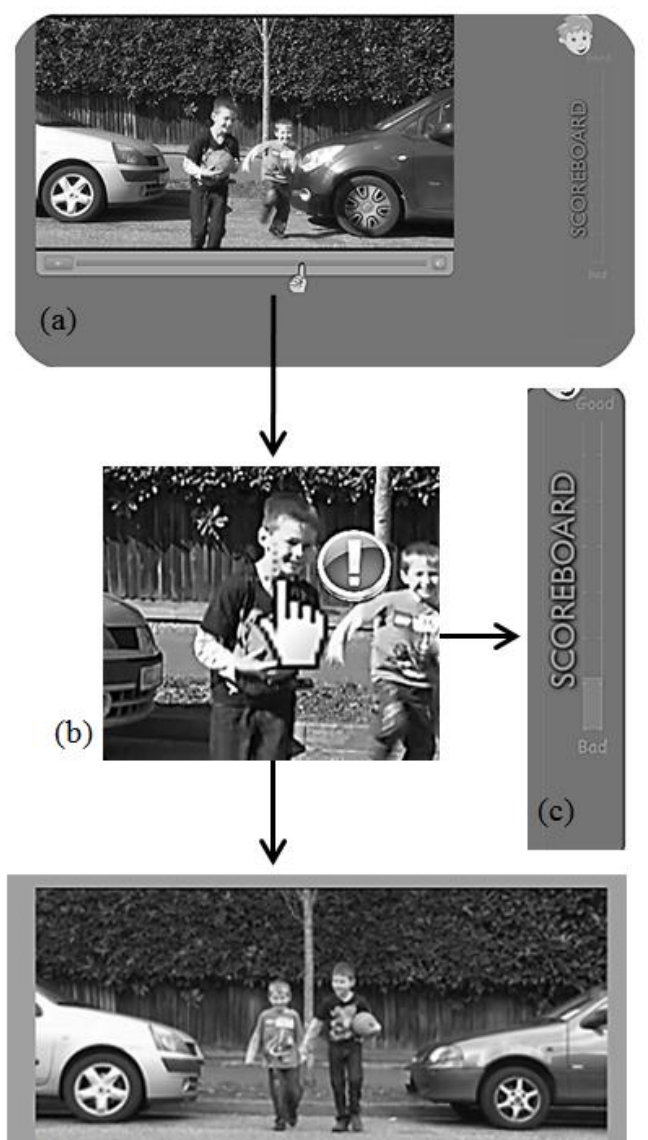

(d)

Fig. 3. The interactive video interface and hazard identification/feedback sequence.

\section{B. Assessing the Impact of Interactive Video on Roadside Behavior}

A before-after analysis was conducted to assess the impact of the interactive video on roadside behavior. Following ethical approval, an average-sized, mixed sex junior school located within an urban area of mixed social and economic advantage agreed to take part in the study. Parental consent was sought from two Year 3 classes $(n=50)$ prior to commencing the study and parents were able to withdraw their children from the study at any time.

The two classes were randomly assigned to experimental and control groups, the former using the interactive video, the latter not. Both groups had their roadside crossing abilities assessed at the start of the trial. The experimental group then played the interactive video. Both experimental and control groups were then re-assessed at the roadside. All assessment was under the full supervision of an adult who was able to stop a child should a dangerous road crossing scenario present itself. The control group took part in exactly the same before and after assessments, but did not use the interactive video (Fig. 4).

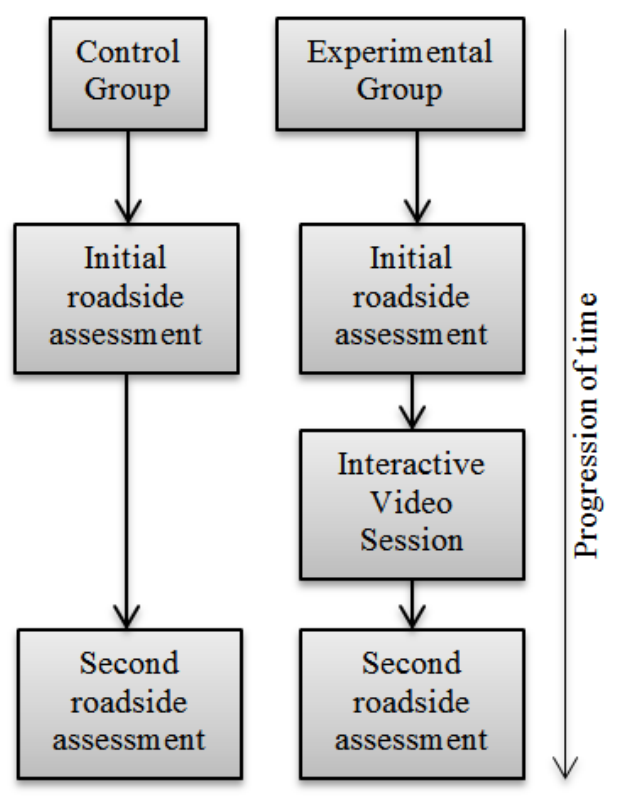

Fig. 4. The study design.

The roadside crossing assessment measured the crossing performance of each child in 6 key areas; stopping at the curb before initiating a crossing, looking for people and clues in cars that might indicate a parked car is ready to move, stopping at the line of sight at the edge of the cars, looking right, left and right again at the edge of the cars, continuing to look after moving from the parked cars and walking safety while crossing. Each area was allocated a score of 0-2 with 2 indicating a complete execution, 1 a partial execution and 0 indicating no attempt to demonstrate a particular skill.

The experimental group was able to use the interactive video with the intention that their understanding of the procedure of crossing between parked cars would improve. Following an introduction to the activity, the progression through the video was self-paced with users spending approximately 20-30 minutes completing the video including the introductory and concluding segments of the video. A researcher was available to assist with any technical issues.
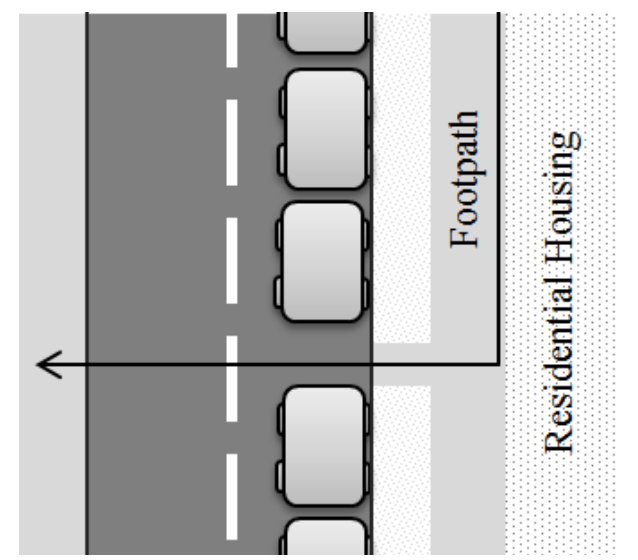

Fig. 5. The configuration of the crossing assessment location.

A safe assessment location was selected close to the school grounds that could be accessed without crossing major roads. The road was a two-lane residential single-carriageway with limited traffic during the day only passing to access houses, 
the school and a small car park adjacent to local shopping facilities. The road was lined with parked cars on one side as a result of unrestricted parking facilities and the pedestrian path was located such that it would force road users to cross between parked cars should they wish to conduct a mid-road crossing. Parking on the other side of the carriageway was restricted, such that it could be ensured that the crossing exit point was always clear and safe with no sight-line obstructions once the crossing was initiated (Fig. 5).

Participants were escorted to the crossing location, in a 1:3 adult to child ratio, under the full supervision of adults. Based on the method employed by Whelen, Towner et al. [5] in the evaluation of the Kerbcraft pedestrian scheme, during the assessment children were asked to hold hands with and then escort an assessor across the road, between the parked cars. Assessors were instructed to resume control of the crossing procedure should any potentially dangerous roadside situation occur. The other assessor observed and recorded the crossing behavior of the children. Both participants and assessors wore high visibility reflective safety jackets and activities were suspended during the first and last thirty minutes of the school day as the roads surrounding the school became busy with cars dropping off and collecting pupils from the participating school and an adjacent school.

Observations also took place during the interactive video sessions and these are reflected on here in order to inform future design decisions.

\section{RESULTS}

\section{A. The Impact of the Interactive Video on Roadside Behavior}

Following random assignment of two classes $\left(n_{\text {total }}=50\right)$ to either the control $(n=21)$ or experimental group $(n=29)$, before and after data on the children's crossing behavior were collected and transcribed (Table I).

Wilcoxon Signed-ranks test suggested that there was no statistically significant difference at the 95\% level $(p=>.05)$ between the experimental and control groups for the skills of "stopping at the curb before moving between the parked cars" and "walking safely while crossing". These skills are not reported further.

TABLE I: AVERAGE SKILL DEMONSTRATION SCORES BEFORE AND AFTER USING AN INTERACTIVE ROAD SAFETY VIDEO (EXPERIMENTAL GROUP)

\begin{tabular}{|l|c|c|}
\cline { 2 - 3 } \multicolumn{1}{l|}{} & \multicolumn{2}{c|}{ Average Skill Score } \\
\cline { 2 - 3 } \multicolumn{1}{l|}{$\begin{array}{l}\text { Stopping at the curb before initiating a } \\
\text { crossing }\end{array}$} & 2.00 & After \\
\hline $\begin{array}{l}\text { Looking for people and clues in cars that } \\
\text { might indicate a parked car is ready to } \\
\text { move }\end{array}$ & 0.00 & 1.00 \\
\hline $\begin{array}{l}\text { Stopping at the line of sight at the edge } \\
\text { of the cars }\end{array}$ & 0.32 & 1.00 \\
\hline $\begin{array}{l}\text { Looking right, left and right again at the } \\
\text { edge of the cars }\end{array}$ & 0.54 & 1.61 \\
\hline $\begin{array}{l}\text { Continuing to look after moving from } \\
\text { the parked cars and walking safety while } \\
\text { crossing }\end{array}$ & 1.46 & 1.82 \\
\hline Walks safely while crossing & 2.0 & \\
\hline
\end{tabular}

In the experimental group the skills of "looking for people and clues in cars that might indicate a parked car is ready to move", "stopping at the line of sight at the edge of the cars", "looking right, left and right again at the edge of the cars" and "continuing to look and remain aware during the crossing", all demonstrated statistically significant improvements in each of the behaviors, $p<.05$ (Table II).

TABLE II: WILCOXON SIGNED-RANKS TEST STATISTICS - EXPERIMENTAL GROUP

\begin{tabular}{|c|c|c|c|c|}
\cline { 2 - 5 } \multicolumn{1}{c|}{} & $\begin{array}{c}\text { Looking for } \\
\text { people and clues } \\
\text { in cars }\end{array}$ & $\begin{array}{c}\text { Stopping at } \\
\text { the line of } \\
\text { sight }\end{array}$ & $\begin{array}{c}\text { Looking right, } \\
\text { left and right } \\
\text { again }\end{array}$ & $\begin{array}{c}\text { Continuing to } \\
\text { look and remain } \\
\text { aware }\end{array}$ \\
\hline $\mathrm{Z}$ & -4.456 & -2.732 & -3.551 & -2.202 \\
\hline $\mathrm{p}$ & .000 & .006 & .000 & .028 \\
\hline
\end{tabular}

Conversely in the control group the same assessed skills set did not demonstrate a statistically significant improvement in each of the behaviors with Wilcoxon Signed-ranks tests indicating that skills did not improve significantly, $p>.05$ (Table III).

TABLE III: WILCOXON SIGNED-RANKS TEST STATISTICS - CONTROL GROUP

\begin{tabular}{|c|c|c|c|c|}
\cline { 2 - 5 } \multicolumn{1}{c|}{} & $\begin{array}{c}\text { Looking for } \\
\text { people and clues } \\
\text { in cars }\end{array}$ & $\begin{array}{c}\text { Stopping at } \\
\text { the line of } \\
\text { sight }\end{array}$ & $\begin{array}{c}\text { Looking right, } \\
\text { left and right } \\
\text { again }\end{array}$ & $\begin{array}{c}\text { Continuing to } \\
\text { look and remain } \\
\text { aware }\end{array}$ \\
\hline $\mathrm{Z}$ & -1.414 & -.414 & -.144 & -1.311 \\
\hline $\mathrm{p}$ & .157 & .679 & .885 & .190 \\
\hline
\end{tabular}

\section{B. The Usability of the Interactive Video and Effectiveness of the Video Design}

Observations on the usability of the interactive video were made during the trial. Issues with the interactive video performance fell into two categories a) sound and b) video lag.

Sound issues were infrequent and related to a participant not being able to hear the voiceover on the introductory elements of the activity. These issues were as a result of a participant inadvertently turning down the in-line remote control on their headphones or inadvertently turning down the computer system volume, either by muting or by reducing the volume using a keyboard shortcut or on-screen action. Due to inexperience, these issues were generally not user-solvable and issues were therefore resolved by the researcher assisting with the activity; turning up the volume on either the in-line remote or on the computer to an acceptable level.

Video lag was experienced when users attempted to access the interactive video by streamed means over the school computer network. This was solved by installing the interactive video locally on each machine however this is a design implication that should be given some consideration.

\section{DISCUSSION}

Baseline performance of "stopping at the curb before initiating a crossing" and "walking safely while crossing" were excellent; indicating understanding of the critical underpinning road safety skills required for safe pedestrian travel throughout life. Baseline performance in other skills specific to parked cars crossing was however minimal; a clear indication that parked cars crossing technique had not been taught to many of the participating children prior to undertaking this activity.

Poor baseline performance, especially in "Looking for people and clues in cars that might indicate a parked car is 
ready to move" and "Stopping at the line of sight at the edge of cars" present a clear safety risk if a child was to independently cross between parked cars as lack of these behaviors in particular would reduce the chance of a child being seen by a driver prior to crossing into the main carriageway. While the majority of children did look for approaching traffic before initiating a crossing, many did not then go on to look right, left and right again at the edge of the cars; another critical skill as the traffic situation may well have changed between stepping off the curb with movement after that moment being obscured from a drivers view.

Following 'training' with the interactive video, the performance of the experimental group showed a statistically significant improvement. By being engaged in an interactive video for between 20-30 minutes, with the core behavioral messages being repeated at least three times, there was a demonstrable improvement in the core skills needed to cross safely between parked cars. Importantly, these behavioral improvements were noticeable after just one session and further exposure to the video may well compound these road safety messages further when accompanied by practical reinforcement.

Despite general improvement of skills, a small number of individual children in the experimental group did not show any improvement in behavior despite using the video. This is an interesting finding that could be a result of a number of factors; in particular it suggests that these users were not engaged in the video and that as a result did not take on board the key safety messages. Or if the users were engaged by the video, they were unable to transfer the video into behavior at the roadside. This highlights the key importance of a blended learning approach where a combination of teaching approaches are likely to have the highest impact on learning [12]; interactive video may not be an effective tool to educate all road users and highlights the importance of it being an addition to practical pedestrian training.

In any adoption of interactive video into a pedestrian training scheme, a number of issues must be taken into account and given consideration.

\section{1) Interactive video is a complementary activity}

Interactive video is intended to be a complementary activity; an addition to practical pedestrian training where there is a need to reinforce learning of a particular skill. It is not designed to be a standalone activity and the results support the need for this to be the case; while behavioral improvement is seen on average across the group, its' effectiveness varies between users and as with all education a range of teaching styles should be implemented depending on the learners.

In this instance, the activity is designed to be classroom based and teacher led such that there is an adult available to rectify any problems and answer questions should any arise.

It is the intention that interactive video could be used alongside or potentially even replace the less effective knowledge-based worksheets that often complement pedestrian training. In a similar amount of classroom based time allocated to paper-based material, users could instead use an interactive video that is designed to target both knowledge and behavioral change; a key desired outcome in a road safety based educational intervention.

\section{2) Interface and computer program design considerations}

While the interactive video did prove to be effective, there is a need to address design issues in future video revisions should they be adopted into a pedestrian training scheme; sound, method of delivery and video content.

Sound will generally be delivered through headphones in a classroom environment; the use of in-line remote controls proved problematic and their use is discouraged in future applications of this type of activity.

Method of delivery is a key consideration. Interactive video can either be streamed (e.g. on a local or public website) or installed locally on a hard drive. While streaming has numerous advantages such as a single installation and central administration capabilities, it requires sufficient bandwidth on school computer networks to be executed effectively. Anecdotal evidence from this study suggests that this and other infant schools may lack the internet, Wi-Fi and LAN network speeds required to effectively make high quality interactive videos available on this basis in all cases. While locally installed interactive videos are a very simple solution to this issue, they limit the ability to easily update and centrally administer the video unless the school possesses over the network installation capabilities. While this is not an issue of detriment to the quality of teaching, it is one that will affect the distribution of the learning activity in schools; with local installations requiring more time to set up, administer and deliver. This would have an associated cost.

While not considered to be an issue in this trial context, there is continuity error in the interactive video used for this study. For example parked cars change between scenes as a result of drivers moving their cars and the number of parked cars on the road also fluctuates. While this does not detract from the learning outcomes of the video, each error must be considered individually and removed if it either becomes distracting to the users or undermines the learning outcomes.

\section{3) Training program design considerations}

Interactive video is not designed to replace practical training and it may therefore be unethical to remove elements of practical training in order to replace them with computer based training. Interactive video could however help where a skill has been previously removed from a training scheme in order to save time or reduce costs. For example where parked cars crossing training has been removed from a pedestrian training scheme, interactive video could be used to supplement training with a short classroom-based session followed up with practical on-street reinforcement sessions. In any case it is recommended that a full evaluation of each training program is conducted in order to ensure effectiveness.

\section{CONCLUSION}

This research sought to assess the effectiveness of an interactive video based road safety training aid designed to complement practical pedestrian training where the facilities or resources do not exist to offer full Kerbcraft training in all pedestrian skills. While the system does show potential to improve skills when used alone, this is not the intention, and all training offered through interactive video should be 
supplemented with practical training as not all users respond to computer based-training.

As with pedestrian training there is also a need to emphasize the fact that pedestrian training of any kind, including the use of interactive videos, does not mean that a child is safe on-street or that they are ready for independent travel. Children should be accompanied by an adult at all times at the roadside, until a time that a parent or guardian deems independent travel of an individual is safe.

Importantly, interactive video is not suggested as a replacement for pedestrian training schemes, but a complementary educational material in much the same way that a road safety worksheet or online road safety cartoon computer game also offers further guidance to students. In this example, a parked cars crossing interactive video must be complemented by on-street reinforcement of the procedure and further training in finding safe routes in order to compound the fact that parked cars crossing should only be considered as a last resort when no other safer alternative is available.

\section{ACKNOWLEDGMENT}

The authors of this paper would like to thank the school staff and local authorities that took part or assisted in the implementation of this research and to the pupils and parents for agreeing to take part.

\section{REFERENCES}

[1] World Health Organization (WHO), Global status report on road safety 2013: supporting a decade of action., 2013, WHO: Geneva.

[2] Department for Transport. (2011). Road Accident Data, 2010 [computer file]. 2011: Road Accident Statistics Branch. Colchester, Essex: UK Data Archive [distributor]. [Online]. Available: http://dx.doi.org/UKDA-SN-6926-1.

[3] Department for Transport, Strategic Framework for Road Safety, London, 2011.

[4] J. K. Thomson, C. S. Whelan, M. Dickenson, L. McBrearty, S. MacLean, A. Motion, and J. Docherty, Kerbcraft: Kerbcraft Training Manual, A Handbook for Road Safety Professionals, 2008, Department for Transport: London.

[5] K. Whelen, E. Towner, G. Errington, and J. Powell, Road Safety Research Report No. 82. Evaluation of the National Child Pedestrian Training Pilot Projects, 2008, Department for Transport: London.

[6] J. Hammond, T. Cherrett, and B. Waterson, "An evaluation of child pedestrian training in the UK: the scope for interactive technologies to aid teaching," in Proc. 43rd Anual Meeting of the Universities Transport Study Group (UTSG), 2010.

[7] Department for Transport. Leaders' notes: Road safety activities for out-of-school groups. [Online]. Available: http://think.direct.gov.uk/education/early-years-and-primary/out-of-s chool-groups/.

[8] E. Fokides and C. Tsolakidis, "Virtual Reality in Education: A Theoretical Approach for Road Safety Training to Students," European Journal of Open, Distance and E-Learning, 2008.

[9] T. Cherrett, G. Wills, J. Price, S. Maynard, and I. Dror, "Making Training More Cognitively Effective: Making Videos Interactive," British Journal of Educational Technology, vol. 40, pp. 1124-1134, 2009.

[10] K. Melson, "11.10. O Interactive traffic training for children in Odense," in Proc. Unpublished Report via email.

[11] J. Hammond, T. Cherrett, and B. Waterson, "Toward safer roadside behavior on the school journey through interactive video training," in Proc. 92nd Annual Meeting of the Transportation Research Board 2013, TRB: Washington.

[12] C. Richardson. (2006). Quick Guide to Blended Learning. [Online]. Available:

http://e-learningcentre.ascensioninternet.co.uk/Resource/CMS/Asset s/5c10130e-6a9f-102c-a0be-003005bbceb4/Resources/QG-blended.p df.

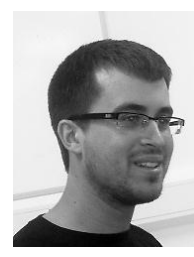

James A. Hammond is an engineering doctorate researcher within the Transportation Research Group at the University of Southampton; having previously graduated with a BEng in Environmental Engineering. His core research interest is road safety; looking in particular at how interactive hazard perception based videos can be used to enhance road safety training.

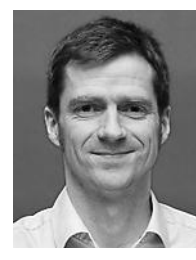

Tom J. Cherrett is a lecturer within the Transportation Research Group, University of Southampton, UK. His areas of specialism related to transport include; Developing sustainable waste disposal strategies and the improving the distribution of goods in urban areas. $\mathrm{He}$ has been involved with several EPSRC and EC projects related to incident detection and journey time estimation using UTC infrastructure. $\mathrm{He}$ is also involved in innovative teaching and learning research looking at how gaming and simulation technologies can be used to promote 'student centered learning' in health, safety and risk education.

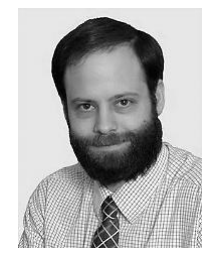

Ben J. Waterson is a member of the Transportation Research Group at the University of Southampton, has background in statistics and operational research methods. His early research focused quantifying and modeling behavioral responses to traveler information. Recently his focus has been on quantifying traffic conditions based on detector data streams, working closely with local authorities to build on research experience within real-time urban journey time estimation, through development incident detection algorithms and enabling research into the fusion of disparate sources of traffic and environmental data to generate more comprehensive views of current network conditions. 